\title{
Automatic Multi-Seed Detection For MR Breast Image Segmentation
}

\author{
Albert Comelli ${ }^{1}$, Alessandro Bruno ${ }^{1}$, Maria Laura Di Vittorio ${ }^{2}$, \\ Federica Ienzi ${ }^{2}$, Roberto Lagalla ${ }^{2}$, Salvatore Vitabile ${ }^{2}$, and Edoardo Ardizzone ${ }^{1}$ \\ ${ }^{1}$ Dipartimento dell'Innovazione Industriale e Digitale (DIID) - Università di Palermo \\ Palermo (PA) - Italy \\ ${ }^{2}$ Dipartimento di Biopatologia e Biotecnologie Mediche - Università di Palermo \\ Palermo (PA) - Italy
}

\begin{abstract}
In this paper an automatic multi-seed detection method for magnetic resonance (MR) breast image segmentation is presented. The proposed method consists of three steps: (1) pre-processing step to locate three regions of interest (axillary and sternal regions); (2) processing step to detect maximum concavity points for each region of interest; (3) breast image segmentation step. Traditional manual segmentation methods require radiological expertise and they usually are very tiring and time-consuming. The approach is fast because the multi-seed detection is based on geometric properties of the ROI. When the maximum concavity points of the breast regions have been detected, region growing and morphological transforms complete the segmentation of breast MR image. In order to create a Gold Standard for method effectiveness and comparison, a dataset composed of 18 patients is selected, accordingly to three expert radiologists of University of Palermo Policlinico Hospital (UPPH). Each patient has been manually segmented. The proposed method shows very encouraging results in terms of statistical metrics (Sensitivity: 95.22\%; Specificity: 80.36\%; Precision: 98.05\%; Accuracy: 97.76\%; Overlap: $77.01 \%$ ) and execution time (4.23 sec for each slice).
\end{abstract}

Keywords: Automatic Segmentation, Breast MR, Maximum Concavity Points, Seed Detection.

\section{Introduction}

Nowadays, medical research focuses on the optimization of the workflow from the acquisition to final report of detected lesions [1] and for volumetric measurements from preoperative staging and evaluation after neo-adjuvant chemotherapy [2]. In last decades the scientific community has shown a growing interest towards the analysis of breast images. As a matter of fact, nowadays the CAD (computer aided diagnostic) systems are representing a second reader to help radiologist in interpretation task. Breast imaging is an effective tool for detection of suspicious regions in breast tissue, contributing to a noticeable decrease of mortality associated with breast cancer [3][4]. Breast MRI is a medical imaging technique used for the analysis of breast tissue. MRI acquires a set of volumetric data with a high contrast between fatty tissue and fibroglandular tissue [5]. This imaging modality is excellent for measurement of volumetric breast density. In last decade several CAD (computer aided diagnostic) methods have been 
developed to allow the radiologists and physicians in advanced analysis and inspection of breast tissue properties such as breast density [6]. Furthermore CAD methods allow to detect breast lesions useful for prevent breast cancer. Breast MRI is increasing its popularity as a screening modality for high-risk patients or patients with dense breasts. A fundamental role in Computer Aided Diagnostic methods is played by segmentation of the breast. A good breast segmentation allows to avoid processing irrelevant features, such as background and the tissue not belonging to the breast regions. A good breast segmentation on MRI confines the CAD systems to focus on the breast tissue, improving the specificity by eliminating false positives outside the ROI (region of interest) i.e. the breast tissue. Generally, the first step in CAD systems is the segmentation of the breast area on MR images, then, the second step is the detection and the exclusion of the chest wall muscle. A key challenge for medical imaging is to measure the volume of fibroglandular tissue and the density in MRI by normalizing to the breast volume. It is not easy to design approaches for automatic breast segmentation because of the large variety of breast in shape and pattern. In more detail, the major issue is to delineate the lateral posterior and the chest wall muscle boundaries. The radiologists observed that in MRI of dense breasts, the visual properties of fibroglandular tissue can be quite similar to the chest wall muscle [7]. Thus, as consequence of the aforementioned remarks, it increases the technical difficulty to delineate and exclude the muscle while preserving the dense breast tissue.

In this paper an automatic multi-seed detection method for magnetic resonance (MR) breast image segmentation is proposed. The method shows very encouraging results compared to the gold standard in terms of statistical metrics (Sensitivity: 95.22\%; Specificity: 80.36\%; Precision: 98.05\%; Accuracy: 97.76\%; Overlap: $77.01 \%)$ and execution time (4.23 sec for each slice). The implementation of the proposed method has been running on a general purpose PC with a 2,3GHz Intel Core i5 processor, 8 GB $1333 \mathrm{Mhz}$ DDR3 memory, and Mac OS x 10.8.5 version. The article is organized as follows: In the 2nd Section the meaningful works on images segmentation are described; In the 3rd Section the breast MRI dataset used for development, test, and evaluation of the proposed system is described; In the 4rd Section, the proposed system is discussed; In the 5th Section depicts results and discussion; The final considerations are treated in the 6th Section.

\section{Related Works}

In breast MRI, several elements are required to perform automatic analysis. Many examples of medical imaging require an initial segmentation phase: multimodal breast image registration, computer aided analysis of DCE (dynamic contrast enhanced) MRI [8], and breast density assessment [5][6]. In [8] the authors detected the left side and the right side of the breast and the center of mass in each side is used as the seed points for region growing. The region detection and extraction from the anatomical regions are very difficult tasks. Complicating factors are the large shape variations of pectoral muscles across different 
patients, the similarity between intensity distributions and texture descriptors of the breast MR in muscle and fibroglandular tissues.

In last few years, many researches appears in medical imaging and precise segmentations of relevant anatomical structures such as breast region and fibroglandular tissue are required. Most of state of the art methods for breast segmentation on MRI are semi or fully automated, furthermore they can be grouped in contour-based, region-based and atlas-based approaches [9]. Generally, on Breast MRI, the following operations precede the breast segmentation task: Pectoralis muscle boundary segmentation, breast-air boundary segmentation, In [10] the authors proposed a method based on the observation that the pectoralis muscle and breast-air boundaries exhibit smooth sheetlike surfaces in 3D. This surfaces which can be simultaneously enhanced by a Hessian-based sheetness filter. The authors in [11]proposed a method for breast segmentation, but it needs manual intervention. In [12] breast segmentation was based on a semiautomated model that accounting for partial volume effects.

In [13]the authors proposed an automatic segmentation method based on the second derivative information represented by the Hessian matrix. Koenig et al. [14]performed a method to detect the most important strutctural elements of the breast by using BI-RADS criteria. Nie et al. [15] proposed a method for the analysis of breast density based on three-dimensional breast MRI: they first performed breast segmentation including an initial segmentation based on body landmarks of each individual woman, then they used fuzzy C-mean classification to exclude air and lung tissue, last they performed B-spline curve fitting to exclude chest wall muscle. Xiaoua et al. [16] proposed a method within a Bayesian framework, based on a maximum a posteriori estimation method. In [17] Guberna-Mérida et al. performed breast segmentation by using a framework based on Atlas (a technique for automatic delineation of anatomical structures in different 3D image modalities). In [18] the authors extended the method [18] by adding a combination of image processing techniques such as signal intensity inhomogeneities correction and probabilistic analysis as Expectation Maximization. In [19] Gallego-Ortiz et al. performed breast segmentation on MRI by combining a 3-D edge detection method with a probabilistic atlas of the breast. In [20] the authors focused the attention on fibroglandular tissue segmentation on Breast MRI: a fully automated segmentation algorithm, to estimate the volumetric amount of fibroglandular tissue in breast MRI. To optimize the computational cost of image segmentations, a lot of approaches are applied. PCA [21], unsupervised classification [22], and fuzzy c-means [23] reduces the dimensionality of the data therefore reduces the computational cost of analyzing new data [21].

\section{Materials}

The dataset consists of 18 patients from UPPH. The patient were divided in two groups according to their age: group 1 ( $25 / 35$ years old, glandular/fibroglandular tissue) and group 2 (45/55 years old, fibrofatty/fatty tissue). A GE signa excite1.5 T HD 23 scanner was used to acquire T1 FSE axial sequences with the 
following technical parameters: 4 channels coil; $\mathrm{TR} / \mathrm{TE}=525$; echo train $=$ $2 ;$ image slices $=40 ;$ slice thickness $=5 \mathrm{~mm}$; slice gap $=0 ; \mathrm{FOV}=160 \mathrm{X} 320$; bandwidth $=41.67 \mathrm{~Hz}$; imaging matrix $=512 \times 256$.

\subsection{Gold Standard}

Three medical doctors, one resident and two radiologists, with progressively increasing knowledge level of breast imaging, performed the manual segmentation by using DICOM viewer Osirix [24] and following these criteria: breast parenchyma and cutaneous surface were isolated from external air basing on its lower intensity; lower boundary of breast region was delimited by using pectoral muscle as landmark; lateral bounds were represented by axillary cavities. The radiologists usually do not agree with each other, then the results from several observers are used to define a consolidated reference to compare the inter-observer variance, as in [25].

\section{Methods}

The proposed method consists of three steps: (1)pre-processing step to locate three regions of interest (axillary and sternal regions); (2)processing step to detect maximum concavity points for each region of interest; (3)breast image segmentation step. Eighteen patients have been manually segmented accordingly to three expert Radiologists to generate Gold Standard ground-truth used to evaluate the effectiveness of the proposed method. The acquisition parameters and characteristics are depicted in the next section. The algorithms used in the proposed segmentation method are briefly described in the next sections.

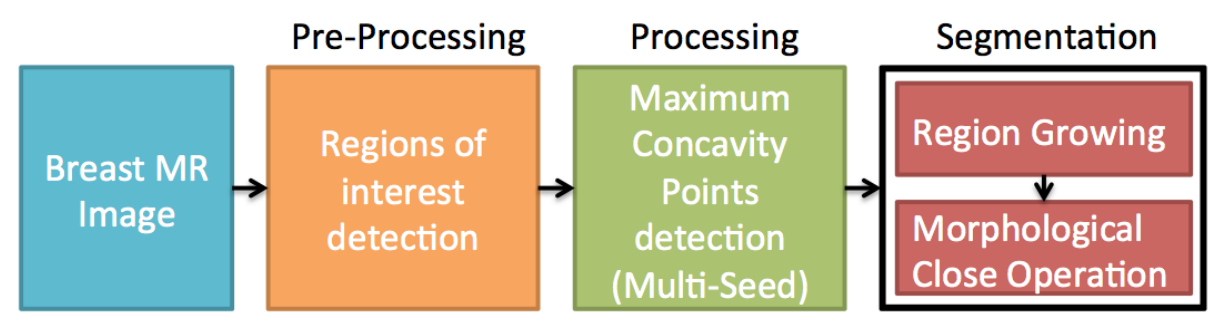

Fig. 1: Block scheme of the Proposed method: Pre-processing, Processing, and Segmentation.

The proposed system consists of three main steps, as depicted in Figure 1:

- The pre-processing output as depicted in Figure 2;

- The processing output as depicted in Figure 3;

- The segmentation output as depicted in Figure 4. 


\subsection{Pre-processing}

The pre-processing step is as follows:

1. A breast MRI study is loaded. It contains, more or less 30 slices with thickness $5.00 \mathrm{~mm}$ (a single slice as shown in Figure 2a);

2. A first binarization step is required to extract the boundary of the breast as shown in Figure 2b, an adaptive thresholding is applied to the image by analyzing the trimodal distribution of intensity histogram;

3. An image crop containing the breast regions is considered (approximatively $2 / 3$ of the whole image) as shown in Figure 2c;

4. The holes are filled in the image, as shown in Figure 2d;

5. The Largest Connected Component is found in the MRI and all other components are removed, as shown in Figure 2e;

6. The objective is to find the coordinates of three pairs of points (A, B, C, D, E, F. as shown in Figure 2f), each pair of points includes a concave region of the breast boundary. The first and the third pair of points correspond to the vertices of axillary regions, the second pair of points correspond to the sternal region. The task is find a n-by-2 matrix that specifies the convex hull including the Breast Region and each row includes the coordinates of the convex hull corners. We notice that the larger side of the convex hull correspond to our regions of interest i.e. the axillary and stern regions (as suggested by the radiologists). In few words we find the three pairs of points by detecting and sorting the larger sides of the aforementioned convex hull. The first three sides of the convex polygon respectively correspond to the axillary (A, B, E, F. see in Figure 2f) and the sternal regions (C, D. see in Figure 2f). We sort the vector including the distances between the consecutive vertices of the convex polygon, in descend order, then we select the first three pairs of coordinates as the vertices of our regions of interest (axillary and sternal regions), as shown in Figure 2f;

7. the convex hull of the binary regions found in step 5 is computed, as shown in Figure 2g;

8. The boundary of image obtained in item 5 is extracted with the canny filter, as shown in Figure 2h;

\subsection{Processing}

The coordinates of the points A-F (see in Figure 2f) are grouped in three pairs: $\mathrm{A}$ and $\mathrm{B}$ belong to the first side of the convex hull (axillary region), C and D belong to the second side of the convex hull (stern region), $\mathrm{E}$ and $\mathrm{F}$ belong to the third side of the convex hull (axillary region). To detect the maximum concavity points (in breast image, see green points G,H,I in Figure 3c) we process three regions of interest i.e. the axillary regions and the stern region. We highlight that the maximum concavity points correspond to the landmarks identified by the radiologists. To accomplish the detection of the maximum concavity points, each region of interest is processed as it follows: 


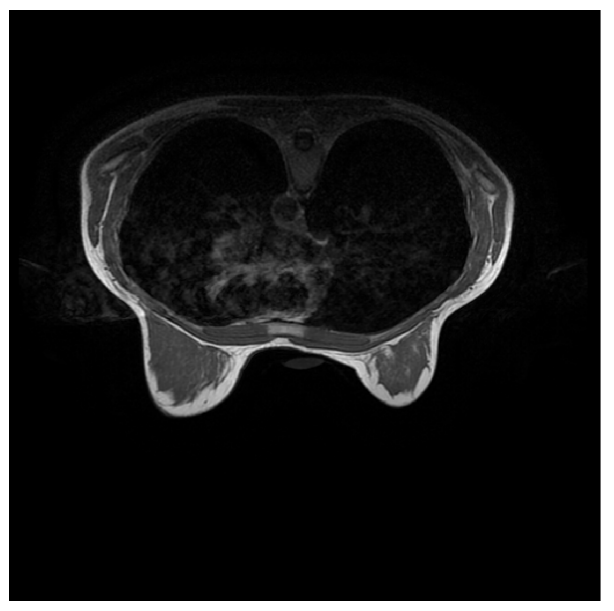

(a)

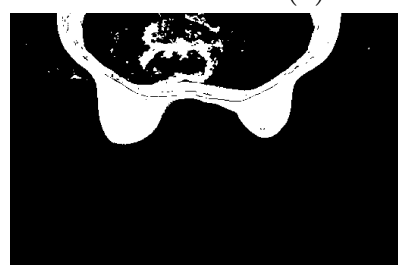

(c)

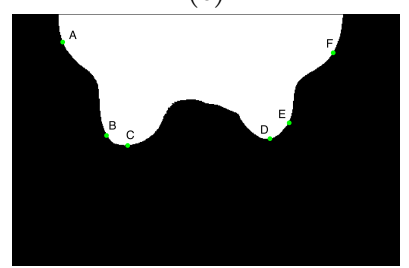

(f)

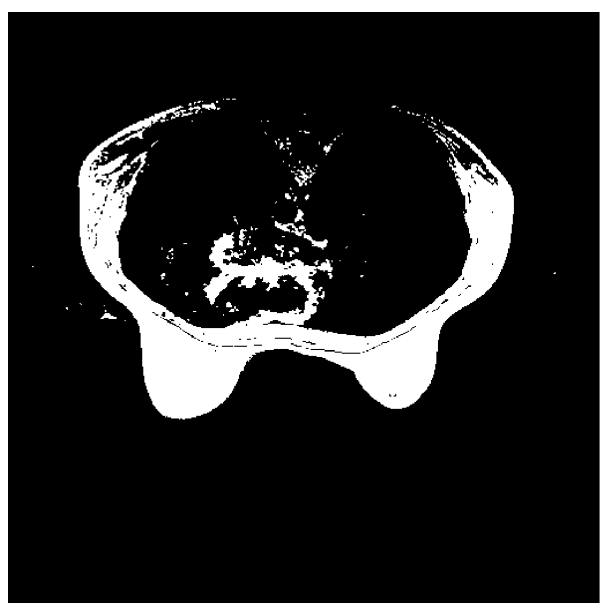

(b)

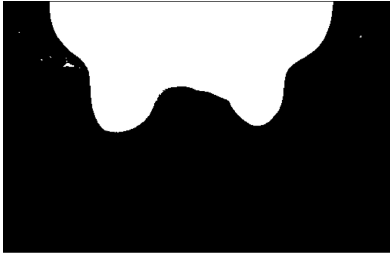

(d)

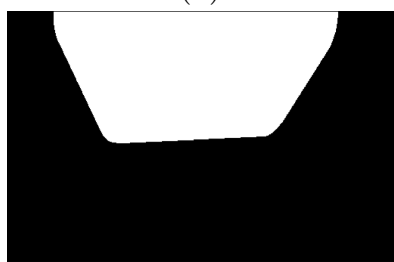

(g)

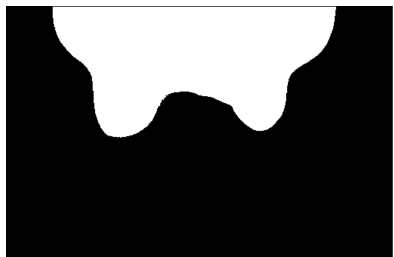

(e)

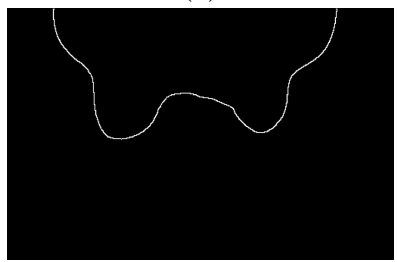

(h)

Fig. 2: Pre-processing: a) A breast MRI is loaded; b) The adaptive thresholding is applied in the MRI to emphasize the contours of the breast; c) image crop containing the breast regions is considered; d) The holes are filled in the image obtained in Figure 2c; e) The Largest Connected Component is found in the MRI and all other components are removed; $f$ ) the coordinates of three pairs of green points (A, B, C, D, E, F.) are found; g) The convex hull is computed in the image obtained in Figure 2e and it is returned a binary convex hull image; h) The boundary of image obtained in Figure 2e is extracted with the canny filter. 
1. To detect the maximum concavity, we first apply Delaunay Triangulation between the two vertices of each region of interest (in first instance A-B in Figure 2f) and all the boundary points between the two vertices (yellow dots in Figure 3a);

2. The area of each triangles is computed;

3 . The triangles are sort in descend order with respect to area value;

4. The first triangle is selected, it includes the larger area in the concave region of interest;

5. The maximum concavity point of the region is the third vertex of the triangle selected in the previous step (see green point G in Figure 3c);

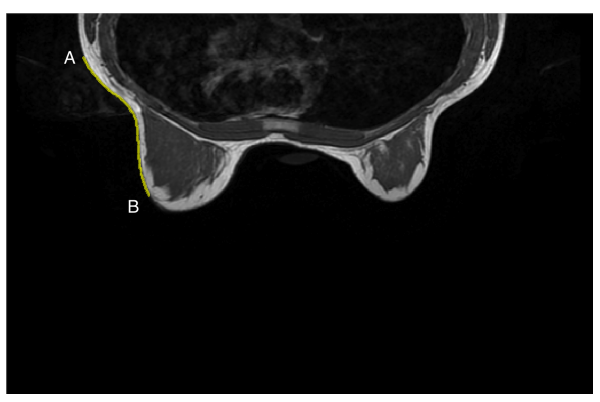

(a)

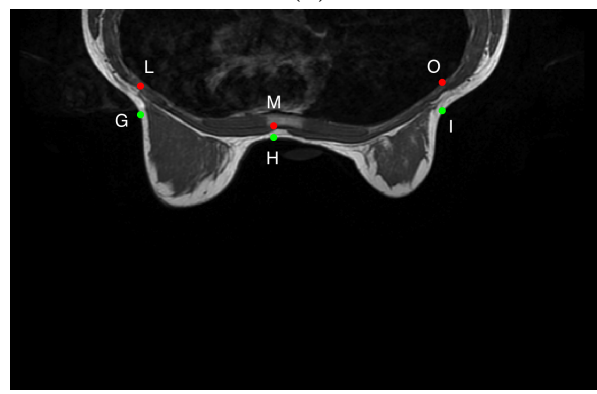

(c)

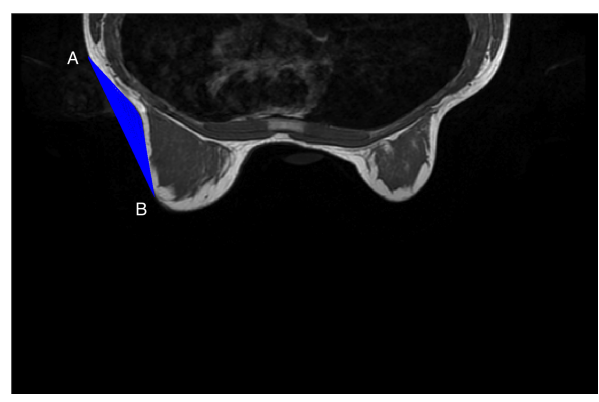

(b)

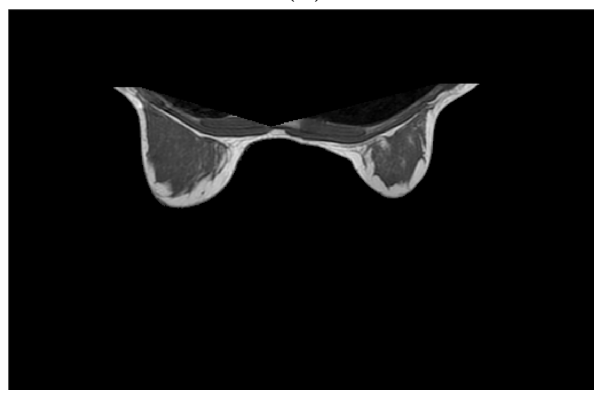

(d)

Fig. 3: Processing: a)The boundary points between the two vertices A-B (yellow dots) are plotted; b) Delaunay Triangulation is applied between the two vertices of each region of interest (in first instance A-B) and all the boundary points between the two vertices (yellow dots in Figure 3a; c) For each concavity To detect the maximum concavity points (in breast image, see green points G,H,I); An additional margin is computed by measuring the vertical distance between $G$ and the inner boundary extracted by filtering with canny algorithm, as depicted by red points in Figure 3c; d) When the extraction of concavity points stage is complete, a line is drawn to join these points. All components above this line are removed.

6. To avoid the exclusion of some region of interest including important features such as Skin, subcutaneous fat pad, and chest fat pad we add and additional margin to the $\mathrm{G}$ coordinates; 
7. The additional margin is computed by measuring the vertical distance between $\mathrm{G}$ and the inner boundary extracted by filtering with canny algorithm. The same technique is applied to the other maximum concavity points, as depicted in Figure 3c by red points;

8. When the maximum concavity points are detected, a line is drawn to join these points. All the elements located above the line are deleted, as shown in Figure 3d.

\subsection{Segmentation}

The segmentation phase consists of three steps:

1. First, a region growing algorithm [26] with standard parameters (threshold) is applied to the image processed as described in the previous section. Region Growing algorithm needs a seed point to be executed. The maximum concavity points are then used as seed points for region growing. The result is shown in Figure 4a;

2. In second step, morphological close operation has been used to fill the holes emerged from region growing. The structuring element of morphological operations is a disk with radius of 20 pixels so that the largest hole gets filled. The disk structuring element is used to preserve the circular nature of the object. The result is shown in Figure 4b;

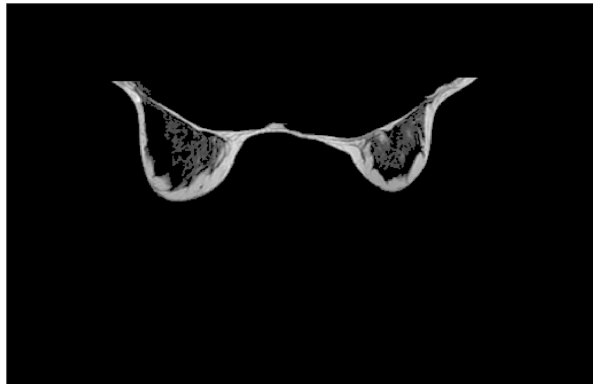

(a)

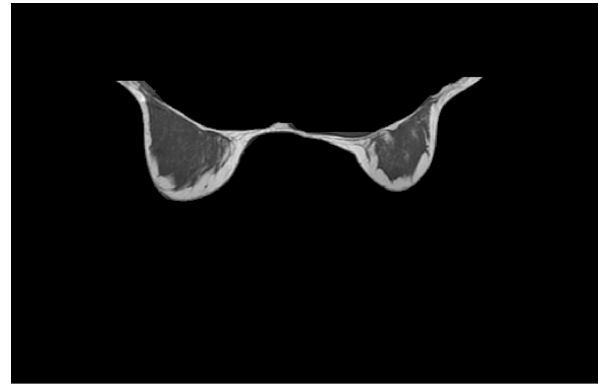

(b)

Fig. 4: Segmentation: a) The region growing is applied to the image obtained in the end of processing step Figure $3 \mathrm{~d}$; b) The holes emerged form region growing are filled by applying the morphological close operations.

\section{Results and Discussion}

The proposed method has been tested on the dataset described in the materials section. The results showed that the proposed method achieves excellent results, as depicted in Table 1. Performance measures are then calculated regarding correct/incorrect segmentation.

The following measures are computed: Sensitivity, Specificity, Negative Predictive Value, Precision, Accuracy, and Error scores: 
- Sensitivity: It is defined as the percentage of effective positives that are correctly identified as such:

$$
\text { Sensitivity : } \frac{T P}{T P+F N}
$$

- Specificity: It is defined as the percentage of effective negatives that are not classified as such:

$$
\text { Specificity : } \frac{T N}{T N+F P}
$$

- Precision: It is defined as, related to reproducibility and repeatability, the degree to which repeated segmentations under unchanged conditions show the same results:

$$
\text { Precision : } \frac{T P}{T P+F P}
$$

- Accuracy: It is defined as the degree of closeness of unsupervised segmentations of a breast to that manual segmentation:

$$
\text { Accurancy : } \frac{T P+T N}{(T P+T N+F P+F N)}
$$

- Overlap: It is defined as the index to quantify agreement between the unsupervised segmentation and manual segmentation:

$$
\text { Overlap : } \frac{T P}{(T P+F P+F N)}
$$

Furthermore, our method has been compared with a state of the art approach $[8]$ to evaluate the effectiveness and the accuracy in breast segmentation. The results are depicted in Table 1. The proposed method shows very encouraging results in terms of statistical metrics (Sensitivity: 95.22\%; Specificity: 80.36\%; Precision: $98.05 \%$; Accuracy: 97.76\%; Overlap: $77.01 \%)$ and execution time (4.23 sec for each slice).

Table 1: Experimental Results

\begin{tabular}{|l|l|l|}
\hline & \multicolumn{2}{|c|}{ Methods } \\
\hline Segmentation & Proposed Method & $\begin{array}{l}\text { Template-Based } \\
\text { Method [6] }\end{array}$ \\
\hline Sensitivity Eq.(1) & $95,22 \%$ & $88,47 \%$ \\
Specificity Eq.(2) & $80,36 \%$ & $78,12 \%$ \\
Precision Eq.(3) & $98,05 \%$ & $92,99 \%$ \\
Accuracy Eq.(4) & $97,76 \%$ & $92,54 \%$ \\
Overlap Eq.(5) & $77,01 \%$ & $73,84 \%$ \\
\hline
\end{tabular}

\section{Conclusions}

In this paper an automatic multi-seed detection method for magnetic resonance (MR) breast image segmentation is presented. The multi-seed detection has been focused because of its importance in regional segmentation technique as the region growing. The maximum concavity points have been proposed as the seed points for the breast MR image segmentation. The detection of this points is based on the identification of three ROI of the breast MR image: the axillary 
regions and the sternal region. The Gold Standard, described in materials section, is used to compute the effectiveness and the performance of the proposed method. The preliminary results are very encouraging in terms of statistical metrics and execution time. In future works we are interested to extend the number of cases study and to develop a CAD (computer aided diagnostic) to detect suspicious regions on breast MRI: the first step is to detect the region of interest by using our proposed method (segmentation phase), than a further analysis and investigation should be conducted to detect suspicious regions by analyzing several features such as texture descriptors, statistical descriptors, histogram of gradients and others state of the art techniques.

\section{References}

1. Giannini, V., Vignati, A., Morra, L., Persano, D., Brizzi, D., Carbonaro, L., Bert, A., Sardanelli, F., Regge, D.: A fully automatic algorithm for segmentation of the breasts in dce-mr images. In: Engineering in Medicine and Biology Society (EMBC), 2010 Annual International Conference of the IEEE, IEEE (2010) 31463149

2. Mann, R.M., Balleyguier, C., Baltzer, P.A., Bick, U., Colin, C., Cornford, E., Evans, A., Fallenberg, E., Forrai, G., Fuchsjäger, M.H., et al.: Breast mri: Eusobi recommendations for women's information. European radiology 25(12) (2015) 3669-3678

3. Giger, M.L., Karssemeijer, N., Schnabel, J.A.: Breast image analysis for risk assessment, detection, diagnosis, and treatment of cancer. Annual review of biomedical engineering 15 (2013) 327-357

4. Roxanis, I., Colling, R.: Use of digital image analysis for outcome prediction in breast cancer. Diagnostic Pathology 1(8) (2016)

5. Brooksby, B., Pogue, B.W., Jiang, S., Dehghani, H., Srinivasan, S., Kogel, C., Tosteson, T.D., Weaver, J., Poplack, S.P., Paulsen, K.D.: Imaging breast adipose and fibroglandular tissue molecular signatures by using hybrid mri-guided nearinfrared spectral tomography. Proceedings of the National Academy of Sciences 103(23) (2006) 8828-8833

6. Patel, B.C., Sinha, G.: An adaptive k-means clustering algorithm for breast image segmentation. International Journal of Computer Applications 10(4) (2010) 35-38

7. Wu, S., Weinstein, S.P., Conant, E.F., Schnall, M.D., Kontos, D.: Automated chest wall line detection for whole-breast segmentation in sagittal breast mr images. Medical physics 40(4) (2013)

8. Lin, M., Chen, J.H., Wang, X., Chan, S., Chen, S., Su, M.Y.: Template-based automatic breast segmentation on mri by excluding the chest region. Medical physics 40(12) (2013)

9. Wang, L., Chitiboi, T., Meine, H., Günther, M., Hahn, H.K.: Principles and methods for automatic and semi-automatic tissue segmentation in mri data. Magnetic Resonance Materials in Physics, Biology and Medicine 29(2) (2016) 95-110

10. Wang, L., Platel, B., Ivanovskaya, T., Harz, M., Hahn, H.K.: Fully automatic breast segmentation in 3d breast mri. In: Biomedical Imaging (ISBI), 2012 9th IEEE International Symposium on, IEEE (2012) 1024-1027 
11. Khazen, M., Warren, R.M., Boggis, C.R., Bryant, E.C., Reed, S., Warsi, I., Pointon, L.J., Kwan-Lim, G.E., Thompson, D., Eeles, R., et al.: A pilot study of compositional analysis of the breast and estimation of breast mammographic density using three-dimensional t1-weighted magnetic resonance imaging. Cancer Epidemiology and Prevention Biomarkers 17(9) (2008) 2268-2274

12. Lee, N.A., Rusinek, H., Weinreb, J., Chandra, R., Toth, H., Singer, C., Newstead, G.: Fatty and fibroglandular tissue volumes in the breasts of women 20-83 years old: comparison of x-ray mammography and computer-assisted $\mathrm{mr}$ imaging. AJR. American journal of roentgenology 168(2) (1997) 501-506

13. Wang, L., Filippatos, K., Friman, O., Hahn, H.K.: Fully automated segmentation of the pectoralis muscle boundary in breast mr images. In: SPIE Medical Imaging, International Society for Optics and Photonics (2011) 796309-796309

14. Koenig, M., Laue, H., Boehler, T., Peitgen, H.O.: Automatic segmentation of relevant structures in dce mr mammograms. In: Medical Imaging, International Society for Optics and Photonics (2007) 65141S-65141S

15. Nie, K., Chen, J.H., Chan, S., Chau, M.K.I., Yu, H.J., Bahri, S., Tseng, T., Nalcioglu, O., Su, M.Y.: Development of a quantitative method for analysis of breast density based on three-dimensional breast mri. Medical physics 35(12) (2008) $5253-5262$

16. Xiaohua, C., Brady, M., Lo, J.L.C., Moore, N.: Simultaneous segmentation and registration of contrast-enhanced breast mri. In: Biennial International Conference on Information Processing in Medical Imaging, Springer (2005) 126-137

17. Gubern-Mérida, A., Kallenberg, M., Martí, R., Karssemeijer, N.: Segmentation of the pectoral muscle in breast mri using atlas-based approaches. Medical Image Computing and Computer-Assisted Intervention-MICCAI 2012 (2012) 371-378

18. Gubern-Merida, A., Kallenberg, M., Mann, R.M., Marti, R., Karssemeijer, N.: Breast segmentation and density estimation in breast mri: a fully automatic framework. ieee journal of biomedical and health informatics 19(1) (2015) 349-357

19. Ortiz, C.G., Martel, A.: Automatic atlas-based segmentation of the breast in mri for 3d breast volume computation. Medical physics 39(10) (2012) 5835-5848

20. Wu, S., Weinstein, S.P., Conant, E.F., Kontos, D.: Automated fibroglandular tissue segmentation and volumetric density estimation in breast mri using an atlas-aided fuzzy c-means method. Medical physics 40(12) (2013)

21. Agnello, L., Comelli, A., Vitabile, S.: Feature Dimensionality Reduction for Mammographic Report Classification. Springer (2016)

22. Comelli, A., Agnello, L., Vitabile, S.: An ontology-based retrieval system for mammographic reports. In: Computers and Communication (ISCC), 2015 IEEE Symposium on, IEEE (2015) 1001-1006

23. Agnello, L., Comelli, A., Ardizzone, E., Vitabile, S.: Unsupervised tissue classification of brain $\mathrm{mr}$ images for voxel-based morphometry analysis. International Journal of Imaging Systems and Technology 26(2) (2016) 136-150

24. Rosset, A., Spadola, L., Ratib, O.: Osirix: an open-source software for navigating in multidimensional dicom images. Journal of digital imaging 17(3) (2004) 205-216

25. Warfield, S.K., Zou, K.H., Wells, W.M.: Simultaneous truth and performance level estimation (staple): an algorithm for the validation of image segmentation. IEEE transactions on medical imaging 23(7) (2004) 903-921

26. Adams, R., Bischof, L.: Seeded region growing. IEEE Transactions on pattern analysis and machine intelligence 16(6) (1994) 641-647 